\title{
Curvature-Driven Lipid Sorting in Biomembranes
}

\author{
Andrew Callan-Jones ${ }^{1}$, Benoit Sorre ${ }^{2,3}$, and Patricia Bassereau ${ }^{2}$ \\ ${ }^{1}$ CNRS UMR 5207, Laboratoire de Physique Théorique et Astroparticules, Université Montpellier II, 34095 \\ Montpellier Cedex 05, France \\ ${ }^{2}$ Institut Curie, Centre de Recherche, Membrane and Cell Functions Group, CNRS UMR 168, \\ Physico-Chimie Curie, Université Pierre et Marie Curie, 75248 Paris Cedex 05, France \\ ${ }^{3}$ Laboratory of Theoretical Condensed Matter Physics, The Rockefeller University, New York, \\ New York 10065 \\ Correspondence: patricia.bassereau@curie.fr
}

It has often been suggested that the high curvature of transport intermediates in cells may be a sufficient means to segregate different lipid populations based on the relative energy costs of forming bent membranes. In this review, we present in vitro experiments that highlight the essential physics of lipid sorting at thermal equilibrium: It is driven by a trade-off between bending energy, mixing entropy, and interactions between species. We collect evidence that lipid sorting depends strongly on lipid-lipid and protein-lipid interactions, and hence on the underlying composition of the membrane and on the presence of bound proteins.

Ipid and protein sorting are key processes Lthat allow eukaryotic cells to maintain membrane homeostasis among organelles during intracellular transport (van Meer et al. 2008). It is known that lipids are not evenly distributed throughout the organelles of the cell. As an example, the percentage of sphingolipids increases along the secretory pathway, going from the endoplasmic reticulum (almost 0\%) to the plasma membrane (about 30\%) (van Meer and Lisman 2002). In the face of continuous lipid trafficking of vesicular intermediates back and forth along this pathway, the cell must be endowed with ways to preferentially sort these lipids to establish and maintain compositional specificity of each organelle.
There is now compelling evidence that sorting occurs during the formation of highly curved transport intermediates (vesicles and tubules). For instance, it has been observed that tubules emanating from endosomes (Mukherjee and Maxfield 2000; Gruenberg 2003; Maxfield and McGraw 2004), as well as vesicles budding from the Golgi apparatus (Brugger et al. 2000; van Meer and Sprong 2004; Klemm et al. 2009), have a lipid composition significantly different from the compartment from which they originate. Mukherjee et al. found that an unsaturated lipid dye was enriched in live cell endosomal tubes, in contrast with a longer chain saturated lipid dye that was excluded (Mukherjee et al. 1999). Furthermore, quantitative analysis of

Editor: Kai Simons

Additional Perspectives on The Biology of Lipids available at www.cshperspectives.org

Copyright (C) 2011 Cold Spring Harbor Laboratory Press; all rights reserved; doi: 10.1101/cshperspect.a004648

Cite this article as Cold Spring Harb Perspect Biol 2011;3:a004648 
the lipid composition of the vesicles formed from the trans-Golgi network showed these vesicles to be enriched in sphingolipids (Klemm et al. 2009), whereas COPI-coated vesicles trafficking from the cis-Golgi network to the endoplasmic reticulum were found to be depleted in sphingolipids (Brugger et al. 2000). These observations, that sphingolipids are depleted from retrograde carriers and are enriched in anterograde ones, are perfectly consistent with the maintenance in the cell of an increasing gradient of this type of lipid along the secretory pathway.

How composition differences between cell organelles are maintained despite intense intracellular lipid exchanges is still poorly understood. Clearly, there is a need for protein and lipid sorting. The case of protein is relatively well explained. For instance, coat proteins are known to specifically bind to transmembrane proteins containing certain peptide sequences, which in turn bind to specific soluble cargoes (Cosson and Letourneur 1994; Stamnes et al. 1995; Barlowe 2000; Ehrlich et al. 2004). The specificity of this type of sorting is largely absent for lipids. Lipids, then, must use other physicochemical means for their sorting:

One way to sort is by the lateral segregation at the nanoscale into domains known as rafts. This very popular hypothesis asserts that proteins that recognize and bind to a given type of raft may form vesicles with the lipid composition of that raft (Simons and Van Meer 1988; Simons and Ikonen 1997; van Meer and Sprong 2004; for a recent review, see Lingwood and Simons 2010). In this mechanism, the lipid composition of the raft is fixed before membrane budding (Fig. 1A).

A second way to sort is by the coupling between lipid composition and the curvature energy of the membrane. Following this mechanism, an inhomogeneously curved membrane could produce an inhomogeneous lipid distribution to minimize the energy cost of bending the membrane (Mukherjee and Maxfield 2000; van Meer and Lisman 2002; Maxfield and McGraw 2004; van Meer and Sprong 2004). Cells can generate inhomogeneous membrane curvature in several ways. For example, COPI or clathrin coats can self-assemble on a membrane surface to form a membrane bud with significantly higher curvature than its surroundings (Antonny 2006). Also, molecular motors walking on microtubules can attach to an intracellular membrane surface and extract a nanoscale-radius tube, which then becomes a transport intermediate (Leduc et al. 2004). The energy cost of bending the membrane into these highly curved shapes depends on the deformability of the bilayer containing different lipids and on the molecular shapes of these lipids. Based on these two membrane properties, lipid sorting between regions of different curvature can reduce the cost of membrane bending. Mathematically, a simple continuum model can be used to describe the coupling between curvature and composition (see Box 1).

The energy needed to bend a patch of membrane of area $A$ is $E=(1 / 2) \kappa\left(C-C_{0}\right)^{2} A$, where $\kappa$ is the bending stiffness, $C$ is the mean curvature, and $C_{0}$ is the spontaneous (relaxed) curvature (see Box 1 for a short introduction to membrane physics). The spontaneous curvature is a local quantity of the membrane, depending on asymmetry between the two leaflets making up the bilayer. Both $C_{0}$ and $\mathrm{\kappa}$ depend on the composition of the curved patch. If this patch is more curved than its surroundings, the bending energy can be lowered by adjusting the composition to reduce $\kappa$ and/or to have $C_{0}$ match $C$. This idea has been explored theoretically, with special emphasis on the interplay between lipid inhomogeneity and the mechanical properties of vesicles (Markin 1981; Seifert 1993; Derganc 2007; Jiang and Powers 2008; Sorre et al. 2009).

The connection between the membrane bending energy and the lipid composition has its origin at the microscopic scale. First, to understand why the notion of spontaneous curvature is related to lipid sorting, consider, for example, a spherical vesicle containing two types of lipids of different shape, one whose head group is wider than its tail, known as inverted-conical (such as lyso-phosphatidic acid (LPA) (Fig. 1B), and another whose head group is roughly as wide as its tail, having a cylindrical shape (such as DOPC) (Fig. 1B). If the outer 


\section{BOX 1. ENERGY OF A TWO-COMPONENT MEMBRANE}

The Helfrich model describes the energy cost of bending a membrane in terms of its curvature (Canham 1970; Helfrich 1973). The Helfrich energy is expressed in terms of two local quantities, the principal curvatures $C_{1}$ and $C_{2}$, as shown in the figure (Fig. B1). They may be positive or negative, and the sign convention is arbitrary. For a closed vesicle or organelle, the principal curvatures are chosen to be positive if the corresponding curves bend toward the inside, and negative if they bend toward the outside.

The bending energy is expressed in terms of the total curvature $C=C_{1}+C_{2}$. For example, the total curvature of a tube is just $C=1 / R$, where $R$ is the tube radius, and of a sphere $C$ $=2 / R$, where $R$ is the sphere radius. The energy per unit area of a bilayer membrane is

$$
f_{b}=\frac{1}{2} \kappa\left(C-C_{0}\right)^{2}
$$

where $\kappa$ is the bilayer bending stiffness and $C_{O}$ is the spontaneous curvature.

The composition dependence of $\kappa$ can give rise to different lipid distributions in the differently curved regions of a vesicle or organelle. Lipid segregation is opposed, however, by loss of mixing energy.

Consider a closed vesicle consisting of two lipid types, 1 and 2, containing a highly curved tube of small area, connected to a large, nearly flat spherical reservoir. The mixing energy penalty per unit area is

$$
\Delta f_{m}=\frac{1}{2} \lambda \Delta \phi^{2}
$$

where $\lambda$ is known as the de-mixing susceptibility; $\Delta \phi=\phi_{t}-\phi_{v}$ and $\phi_{t}$ and $\phi_{v}$ are the fractions of lipid 1 in the tube and reservoir, respectively (the fractions of lipid 2 are given by $1-\phi_{t}$ and $\left.1-\phi_{v}\right)$. The susceptibility is given by

$$
\lambda=\frac{k T}{a}\left(\frac{1}{\phi_{v}\left(1-\phi_{v}\right)}-2 \chi\right)
$$

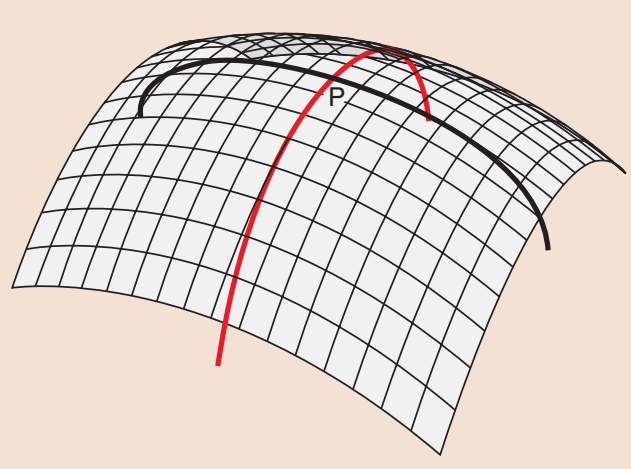

Figure B1. The principal curvatures at point $\mathrm{P}$ are the inverses of the radii of the two circular arcs drawn above. Arbitrarily close to point P the two arcs lie on the surface, but deviate from it moving away from P. Of all possible circular arcs passing through $P$, the arcs of principal curvature are the ones with maximal and minimal curvature.

where $a$ is the area per lipid and $\chi$ measures the strength of short-range lipid-lipid interactions. The first term in parentheses in Eq. (3) represents the entropic "stiffness" for de-mixing; it is largest when $\phi_{\nu}$ is close to zero or one, because then it is highly improbable that very few lipids of type 1 or of type 2 will spontaneously corral together in a small region. Note, however, that this corralling is considerably easier if one of the lipid species is found in the form of clusters. A positive value of $\chi$ means that type 1 lipids prefer to be among each other, as do type 2 lipids, which has the effect of reducing $\lambda$. In fact, as $\chi$ increases, $\lambda$ vanishes for $\phi_{v}=0.5$ and $\chi=2$; this is the critical de-mixing point. For larger values of $\chi$, a uniformly mixed vesicle with $\phi=0.5$ can lower its mixing energy by spontaneously phase separating into lipid-1-rich and lipid-2rich domains of sizes much greater than that of an individual lipid. 
A. Callan-Jones et al.

A

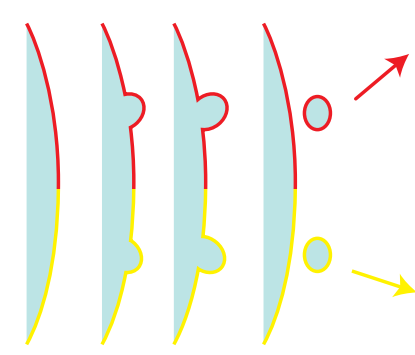

B

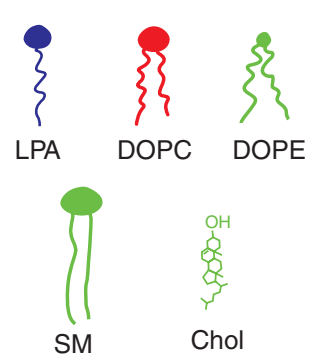

C
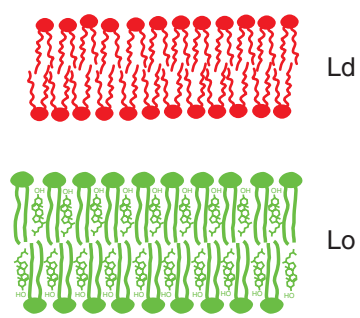

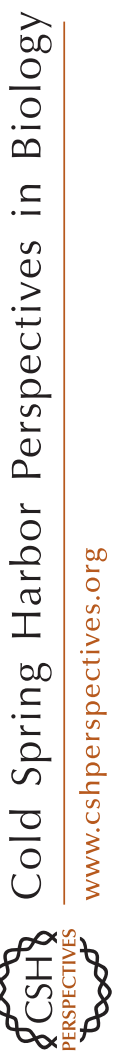

D

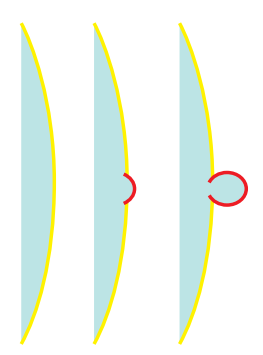

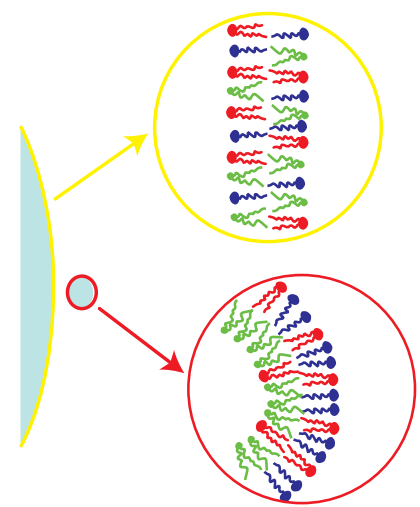

E

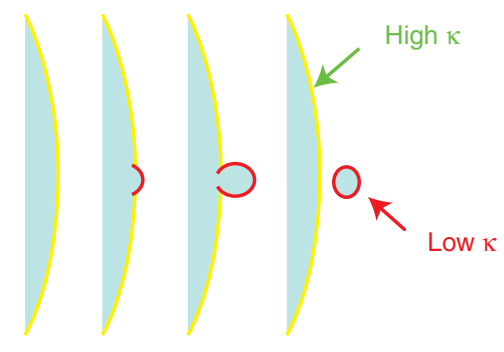

Figure 1. Possible physico-chemical mechanisms for lipid sorting. (A) Lipid sorting based on the raft hypothesis. The composition and destination of newly formed vesicles are determined by the type of raft from which the vesicle originates. $(B)$ Different lipid species and their shape. Lipids are characterized by a shape based on the size of the head group, the number of chains, and the degree of chain saturation. From left to right the shapes are inverted-conical for Lyso-Phosphatidic acid (LPA), cylindrical for unsaturated 1,2-dioleoyl-sn-glycero-3phosphocholine (DOPC), and conical for 1,2-dioleoyl-sn-glycero-3-phosphoethanolamine (DOPE). Bottom: saturated cylindrical Sphingomyelin (SM) and cholesterol. (C) Two physical states relevant for cellular membranes. Top: drawing of a liquid-disordered $\left(\mathrm{L}_{\mathrm{d}}\right)$ membrane, which is rich in unsaturated lipids (with little chain order); bottom: drawing of a liquid-ordered $\left(\mathrm{L}_{\mathrm{o}}\right)$ membrane, which is rich in saturated lipids (with high degree of chain ordering) and cholesterol. (D) Lipid sorting based on spontaneous curvature. The membrane consists of cylindrical, inverted-conical. and conical lipids. The outer leaflet of the budding region is enriched in cylindrical and inverted conical lipids, whereas the inner leaflet is enriched in conical ones. (E) Lipid sorting based on composition dependence of the bending stiffness. The highly curved budding region is enriched in unsaturated lipids, so that the bending stiffness of this region is lower than the surrounding membrane.

leaflet is richer in the inverted-cone lipids and the inner leaflet is richer in the cylindrical lipids, then the vesicle will have a positive spontaneous curvature. Conversely, one would expect that the two leaflets of a spherical vesicle would readjust their composition so that $C_{0}$ can match the curvature of the vesicle (Leibler 1986; Gruner 1989; Kozlov and Helfrich 1992; Seifert 1993). In this case, a transverse asymmetry (i.e., across the leaflets) occurs in the lipid composition, with the outer leaflet being relatively enriched in inverted-conical lipids (Fig. 1D).
In addition, sorting of lipids within a vesicle containing compartments of varying degrees of curvature can occur because of the composition dependence of the bending stiffness (Szleifer et al. 1988; Rawicz et al. 2000; Marsh 2006; Pan et al. 2008; Reeves et al. 2008; Fig. 1E). As the bending stiffness is an elastic property of a patch of membrane, this effect cannot be understood based on individual lipid geometry. Generally speaking, bending a thicker membrane causes greater differential strains between the head and tail groups of a single leaflet than it 
does in a thinner leaflet, and therefore a thicker membrane has a higher bending stiffness. Modeling and experiments confirm that the bending stiffness depends in particular on the square of the bilayer thickness (Evans 1974). Ternary mixtures containing cholesterol, saturated and unsaturated lipids are known to display coexistence of a saturated lipid-rich liquid-ordered $\left(\mathrm{L}_{\mathrm{o}}\right)$ phase and an unsaturated lipid-rich liquid-disordered $\left(\mathrm{L}_{\mathrm{d}}\right)$ phase (Dietrich et al. 2001; Veatch and Keller 2002; Baumgart et al. 2003; Goni et al. 2008) (Fig. 1C). From AFM measurements (Yuan et al. 2002; Lawrence et al. 2003; Connell and Smith 2006), it has been shown that $\mathrm{L}_{\mathrm{o}}$ membranes are thicker than $\mathrm{L}_{\mathrm{d}}$ membranes, and therefore stiffer, as has been measured by tube pulling experiments (Cuvelier et al. 2005; Roux et al. 2005) and pipette aspiration (Rawicz et al. 2008). Typical values of $\kappa$ of ternary vesicles in the $L_{o}$ phase are 50-60 kT, whereas in the $\mathrm{L}_{\mathrm{d}}$ phase, typically 20-30 kT. Here, $k$ is Boltzmann's constant and $T$ is room temperature, such that $k T=4.2$ $\times 10^{-21}$. It was suggested that when a uniformly mixed membrane is subject to inhomogeneous curvature, the bending energy can be reduced by enriching the highly curved regions in the lipids that predominate in the $\mathrm{L}_{\mathrm{d}}$ phase of phase separated membranes (Mukherjee and Maxfield 2000; van Meer and Lisman 2002; Maxfield and McGraw 2004; van Meer and Sprong 2004) (Fig. 1E).

Although the simple picture of curvatureinduced sorting of lipids based on molecular geometry (steric effects) is visually quite appealing, experiments and theory have shown that this is not an effective driving mechanism against the homogenizing effect of entropy (Safran et al. 1990, 1991; Derganc 2007; Mukhopadhyay et al. 2008; Sorre et al. 2009). This result is quite surprising given the widespread view in the past that curvature was a likely candidate to sort lipids based on their shape (Lodish et al. 2003) (Fig. 1D). A simple order of magnitude calculation highlights the importance of entropy. The gain in bending energy in transferring a sphingolipid from a tube of radius $R=20 \mathrm{~nm}$ to a flat reservoir, given a liberal estimate of the difference in bending stiffness between the tube and reservoir of 40 $k T$, is $\Delta E=(1 / 2)\left(\Delta \kappa / R^{2}\right) a=(1 / 40) k T$, for an area per lipid equal to $a=0.5 \mathrm{~nm}^{2}$. We see that this energy is much smaller than $k T$, the scale of thermal energy, meaning that entropy will win out over any gain made in making the transfer. The small value of $\Delta E$ is because of the small size of the lipid dimension compared with the radius of the tube. The above calculation is valid, however, only if we consider the membrane to be an ideal solution of lipids. Membranes consisting of a mixture of lipids are characterized not only by elastic bending but also by the short-range interactions among the different lipid species (see Box 1). Below the miscibility temperature, these interactions give rise to large-scale domains, as seen, for example, by the coexistence of $\mathrm{L}_{\mathrm{o}}$ and $\mathrm{L}_{\mathrm{d}}$ phases (Bagatolli and Gratton 2000; Dietrich et al. 2001; Veatch and Keller 2002; Baumgart et al. 2003; de Almeida et al. 2003; McConnell and Vrljic 2003).

Similar to the bulk behavior of a binary solution, the thermodynamic behavior of mixture membranes results from a competition between entropy and enthalpy (interactions). At high temperatures, thermal fluctuations tend to smooth out compositional heterogeneities, whereas at lower temperatures, depending on the overall composition of the membrane, favorable interactions between like species give rise to domain formation and phase coexistence (see Box 1). Conversely, phase separation can be induced at fixed temperature by adjusting the fractions of cholesterol and saturated and unsaturated lipids (Bagatolli and Gratton 2000; Dietrich et al. 2001; Veatch and Keller 2002; Baumgart et al. 2003; de Almeida et al. 2003; McConnell and Vrljic 2003). This is the more biologically relevant case. As domains have been observed in model membranes mimicking the cell membrane, it is therefore also expected that entropy and interactions play important roles in curvature-induced lipid sorting.

Motivated by an increasing amount of experimental evidence that lipid sorting occurs in cells, there has been a renewed interest recently to develop model membrane systems that can be used to quantify the effectiveness 
A. Callan-Jones et al.

of curvature-based sorting and to compare with theoretical predictions. In this review, we focus on recent works that have been possible because of the convergence of biological interest in lipid sorting and technological advances that have allowed its measurement in vitro. We examine below a number of works that have quantitatively measured the importance that cooperative behavior has on curvature-induced lipid redistribution. Some have considered lipid sorting caused by differences in spontaneous curvature between lipids, whereas others have considered sorting caused by the dependence of bending stiffness on sorting. The overriding conclusion of these works was that curvature is generally ineffective in sorting lipids because of prohibitive entropy costs, yet will be significantly amplified if the membrane composition is tuned to maximize the importance of lipidlipid interactions.

\section{LIPID SORTING REQUIRES LIPID-LIPID INTERACTIONS}

The dependence of the bending stiffness on composition causes lipid sorting between membrane regions of different curvature. Furthermore, lipid-lipid interactions favor like lipids to aggregate. Both effects tend to segregate lipid species and thus act cooperatively to produce significant sorting. In fact, a simple calculation based on a two-component model of the membrane (see Box 1) shows that the degree of sorting is 20 times greater if lipid-lipid interactions are such that the system is only $5 \%$ away from the critical point than if there are no interactions at all. This highlights the importance of lipid-lipid interactions and proximity to phase separation for lipid sorting.

Roux et al. reported the first results on the effect of curvature on lateral lipid organization in vesicle-tubule model systems (Roux et al. 2005). Their work led to two important findings. In the first part of their study, they showed that tubes pulled from $\mathrm{L}_{\mathrm{o}}-\mathrm{L}_{\mathrm{d}}$ phase-separated vesicles by molecular motors were found to be almost exclusively in the $\mathrm{L}_{\mathrm{d}}$ phase. This finding agreed with theory stating that a smaller force is needed to pull a tube with lower bending stiffness. This demonstration showed that collective behavior, here indicated by the existence of domains, was a sufficient condition for curvature-induced lateral organization. In a second part to their work, Roux et al. (2005) also found evidence of curvature-induced lipid sorting in homogenous $\mathrm{L}_{\mathrm{d}}$-phase vesicles. By using two different fluorescent dyes that partition differently in $L_{o}$ and $L_{d}$ domains, they observed that the relative fluorescence of the two dyes was not the same in the tube and in the vesicle. This indicated that a curved membrane could have a different composition than the homogeneous membrane from which it originated. In another study, Parthasarathy et al. studied lateral segregation of lipid domains in inhomogeneous membranes using supported bilayers (Parthasarathy et al. 2006). Here, a substrate was etched to have a periodically modulated ("bumpy") curvature, onto which a membrane was laid. On deposition, the membrane was observed to undergo phase separation in $\mathrm{L}_{\mathrm{o}}$ and $\mathrm{L}_{\mathrm{d}}$ domains, with the $\mathrm{L}_{\mathrm{o}}$ domains preferentially nucleating and localizing in the regions of low curvature. They found, however, that alignment of domains along the weakly curved parallel ridges only occurred below a threshold minimum curvature, indicating the balance between gain in bending energy in aligning versus the entropic tendency to randomize the domains. The results of this paper were in agreement with those of Roux et al. (2005), indicating that a strong spatial gradient of membrane curvature is able to sort $\mathrm{L}_{\mathrm{o}}$ and $\mathrm{L}_{\mathrm{d}}$ domains.

More recently, the works of Sorre et al. (2009) and Tian et al. (2009) quantitatively measured the importance of lipid interactions on sorting using a set-up consisting of a micropipette used to hold a ternary mixture GUV, from which a tube was pulled and held in place either by an attached bead in an optical trap (Sorre et al. 2009) or by a second micropipette (Tian et al. 2009) (Fig. 2). As originally developed by E. Evans (Waugh and Evans 1979), the aspiration pressure, controlled hydrostatically, fixes the tension in the membrane, a key quantity in determining the radius of the tube and the force needed to pull on it; the greater the tension, the narrower the tube. Optical 

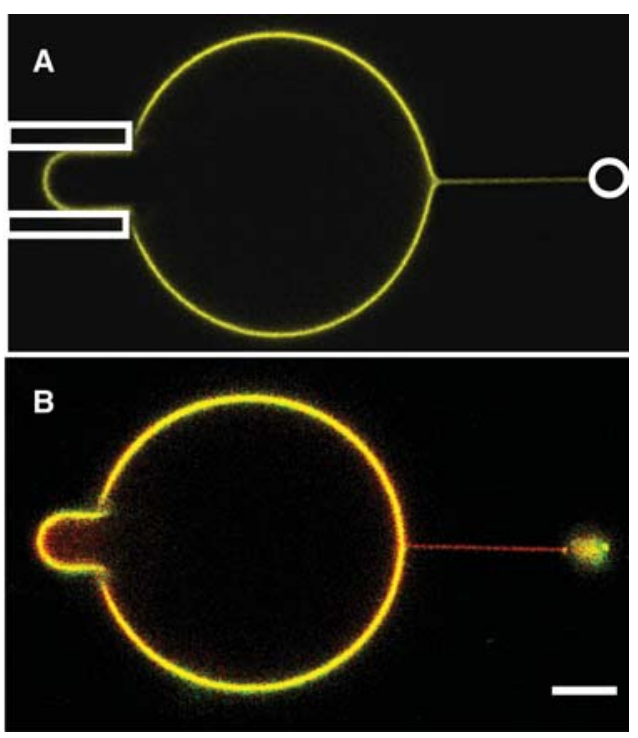

Figure 2. Tube pulling and fluorescence images. $(A)$ A nanoscale-radius tube is extracted from a micronscale GUV that is in turn held by aspiration in a micropipette (left side of image). The tube is held by a Polystyrene bead in an optical trap (indicated by white circle). Here, the membrane composition is far from phase separation. Confocal fluorescence shows that the dye compositions in the tube and GUV are the same. (B) The membrane composition is close to phase separation (BSM:Chol:DOPC 30:35:35 mole\%). Quantitative fluorescence measurement shows a 2.9-fold relative enrichment of the red (Texas red DHPE) over green (BodipyFLC5-GM1) in the tube relative to the GUV. Scale bar, $5 \mu \mathrm{m}$.

trap or micropipette tube pulling experiments provide numerous advantage over experiments on small vesicles (Fig. 2), or even those on tubes pulled by motor proteins. In these experiments, the curvature of the tube can be controlled continuously by adjusting the membrane tension, and the mechanical properties of the membrane can be quantified using the force needed to pull the tube. Moreover, although tubes are rather narrow, their long length ensures that sufficient fluorescence signal can be detected in sorting experiments. Tubes pulled from large vesicles mimic the in vivo situation in which a narrow tether is often extracted from a relatively flat organelle. Furthermore, although small spherical vesicles can only be used to measure differential composition across the bilayer, as might result because of spontaneous curvature differences of lipids, tubes can be used to probe curvature sorting because of both spontaneous curvature and the compositional dependence of the bending stiffness. In this way, the high curvature contrast with the GUV provides an excellent means to test the hypothesis that curvature gradients can sort lipids.

Sorre et al. measured the importance of collective effects on sorting using an integrated tube pulling and confocal imaging setup (Sorre et al. 2009) (Fig. 2A). This setup allowed two ways to detect sorting: by fluorescence microscopy and by pulling force measurement. The GUVs used contained the saturated lipid sphingomyelin (SM), cholesterol, and the unsaturated lipid DOPC, as representative of the plasma membrane. The compositional difference between the tube and vesicle was measured using the partitioning behavior of two types of fluorescent probes. One probe used, Texas Red, is known to preferentially partition into the $\mathrm{L}_{\mathrm{d}}$-phase of a phase-separated vesicle, whereas the other used, a fluorescent GM1 $\left(\mathrm{GM}^{*}\right)$, partitions roughly equally between $L_{d}$ and $L_{o}$ phases. An enrichment of Texas Red relative to $\mathrm{GM}^{*}$ in the tube over that in the vesicle would then be interpreted as a measure of the relative enrichment of unsaturated over saturated lipids in the tube, consistent with earlier observations that stiffer saturated lipid-rich $\mathrm{L}_{\mathrm{o}}$-phase domains are expelled from tubes in phase separated vesicles (Roux et al. 2005). This is exactly what was observed, but not for arbitrary mixtures of the three lipid types: proximity to phase separation was critical for observing sorting. In fact, the amount of lipid sorting was found to increase dramatically near phase separation: as shown in Figure 3A, the sorting value was equal to one, independent of curvature, for a SM fraction up to $15 \%$ (a value of one corresponds to identical compositions in the tube and in the vesicle), whereas it increased strongly with curvature when the SM fraction was equal to $30 \%$, beyond which phase separation occurs. A simple theoretical model, treating the membrane as a binary 
A. Callan-Jones et al.

A

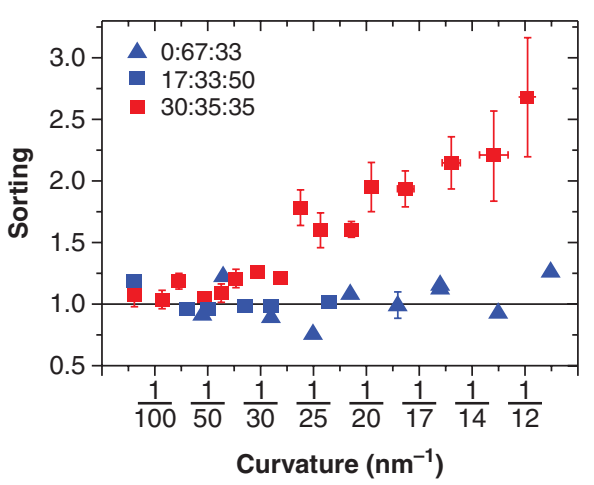

C

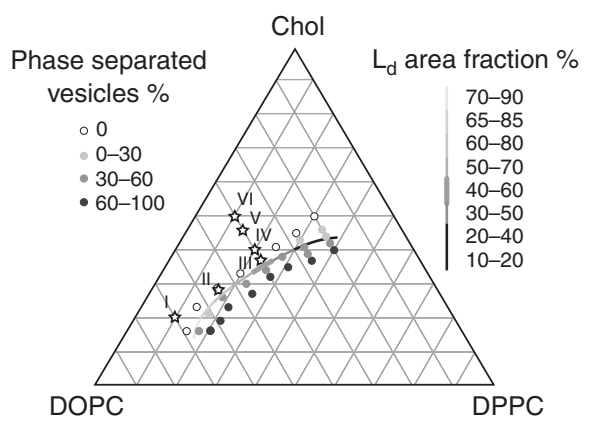

B

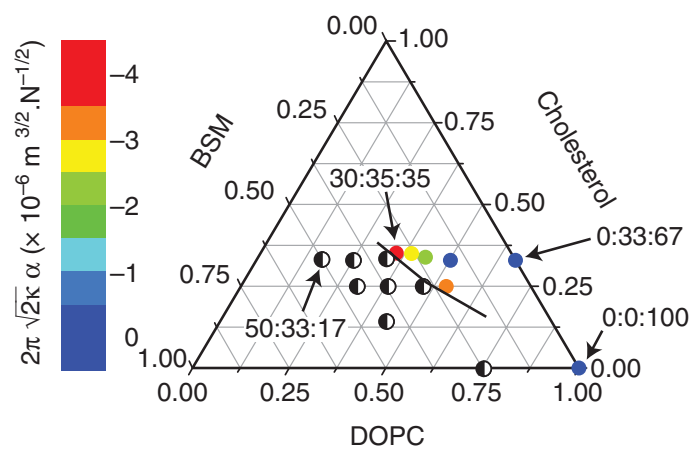

D

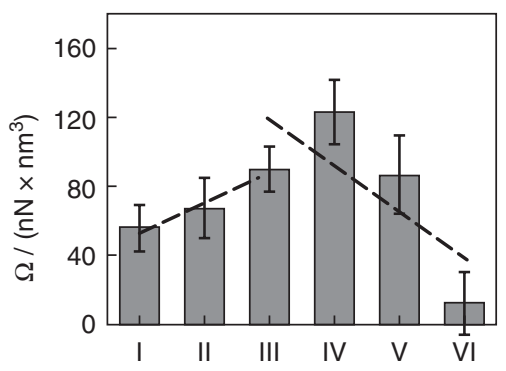

Figure 3. Lipid sorting increases near phase separation. (A) Lipid sorting versus tube curvature, as measured by the partitioning of fluorescent lipid dyes. No sorting is observed for two compositions far from phase separation (the ratio BSM:Chol:DOPC equal to 0:67:33 and 17:33:50). Sorting increases with curvature for a composition near phase separation (30:35:35). Panel adapted from and replotted with express permission from Sorre et al. (2009). (B) The mechanical sorting parameter $2 \pi \sqrt{2 \kappa} \alpha$ increases when the membrane composition approaches the demixing region of the phase diagram of the BSM:Chol:DOPC mixture. Panel adapted from Sorre et al. (2009) and reprinted with express permission from the author. $(C, D)$ The curvature dependence of $\kappa_{e f f}$, indicated by the parameter $\Omega$, also increases for compositions close to phase separation. The lipid mixture used was DPPC:Chol:DOPC. Compositions indicated by numerals I through VI on the phase diagram in $C$ correspond to the histogram bins in $D$. Panels $C$ and $D$ adapted from Tian et al. (2009) and reprinted with permission from Elsevier (C) 2009.

mixture (the fraction of cholesterol was held roughly constant in the experiments), found that the amount of sorting should increase quadratically with tube curvature, in agreement with experiment (Sorre et al. 2009). It is important to note that sorting measured here could only be because of a dependence of bending stiffness on composition, and not to spontaneous curvature. This follows because the measured fluorescence was the signal integrated over the bilayer, and could not distinguish the difference in signal between the two leaflets.
The measurement of the pulling force on the tube provided a second way to assess the coupling between lipid sorting and the energy cost of membrane bending. The force on a tube that has the same composition as the mother vesicle is given by the classical expression $f=2 \pi \sqrt{2 \kappa \sigma}$ (Hochmuth and Evans 1982; Waugh et al. 1992; Evans and Yeung 1994; Derényi et al. 2002; Powers et al. 2002), where $\sigma$ is the membrane tension. Thus, a plot of $f$ as a function of $\sqrt{\sigma}$ should be linear with a slope proportional to $\sqrt{\kappa}$; this relationship 
has been confirmed experimentally, and used as a means to measure bending stiffness. Things are not quite as simple if the compositions on the tube and on the vesicle are different. In fact, for those membrane compositions for which sorting was detected by fluorescence, a clear departure of the dependence of force on tension from the classical law was observed. Intuitively, one expects that at very low tension, for which sorting is small, the force would be given by the usual law; this is what was indeed measured, allowing a measure of $\kappa$, the bending stiffness of the vesicle. As the tension increases, however, the tube becomes richer in lipids forming a more flexible membrane, and hence a reduction relative to $2 \pi \sqrt{2 \kappa \sigma}$ of the force should be observed. This was found experimentally and confirmed by a model (Sorre et al. 2009); theoretically, the effect of sorting on the force was found to follow $2 \pi \sqrt{2 \kappa \sigma}(1-\alpha \sigma)$, and experimentally the sorting parameter $\alpha$ was found to increase monotonically as the region of demixing was approached on the ternary phase diagram (Fig. 3B).

Tian et al. (2009) used various mixtures of unsaturated DOPC, Chol, and saturated DPPC in their tube-pulling experiments, before which they had mapped out the phase diagram to study the effect of proximity of a given GUV composition to phase separation on lipid sorting. For a single component vesicle, or one in which the lipid composition is everywhere homogeneous, the radius of the tube is given by $R=\sqrt{(\kappa / 2 \sigma)}$. Instead of measuring the force, Tian et al. measured the tube radius, independently of the tension, by measuring the change in vesicle projection in the pipette when the length of the tube is varied. They found that $R$ follows the well-known square root law with tension for all compositions, and consequently the bending stiffness is independent of curvature, except when the composition is very close to phase separation. Near phase separation they found that the effective bending stiffness, as defined as $\kappa_{e f f}=2 \sigma R^{2}$, decreased because of sorting, following the law $\kappa_{\text {eff }}=\kappa_{0}-\Omega / R^{2}$, where $\kappa_{0}$ is the bending stiffness of the vesicle and $\Omega$ is a parameter than increases as phase separation is approached
(Fig. 3C,D). Although the techniques and measurements were quite different, the results of Tian et al. (2009) are equivalent to those obtained by Sorre et al. (2009).

A second class of experiment aimed to measure curvature-induced transverse asymmetry based on the spontaneous curvature of different lipid species. Kamal et al. recently measured the transverse distribution of cylindrical, conical, and inverted-conical fluorescent marker lipids in spherical egg-PC vesicles of radii ranging from $10-100 \mathrm{~nm}$ (Kamal et al. 2009). Based on steric effects, they expected to see that the outer leaflet of small vesicles would be enriched in inverted-conical lipids, whereas the inner leaflet would be enriched in conical lipids (Fig. 1D). They did observe some asymmetry in the transverse distribution of their lipid markers, from which they were able to measure the spontaneous curvature of the marker, but found that the asymmetry in the distribution on the two leaflets only reached a maximum of about $20 \%$ for very small vesicles, of $20 \mathrm{~nm}$ in radius. Furthermore, they observed that the asymmetry in the distribution was negligible for vesicle radii greater than $50 \mathrm{~nm}$. Importantly, they considered a dilute amount of labeled lipids to study. As in any dilute system, the effects of lipid-lipid interactions are very weak relative to the entropy of mixing, and hence will not be able to boost the curvature-induced lipid composition asymmetry. Their work was consistent with the negligible asymmetry in lipid marker composition that Tian and Baumgart measured on highly curved tubes pulled from vesicles far away from phase separation (Tian and Baumgart 2009). The key conclusion from these works was that lipid shape alone is not enough to drive measurable lipid sorting; lipid-lipid interactions or lipid-protein interactions are necessary to amplify the curvature-based sorting. This is consistent with calculations on surfactant systems, showing that sufficiently strong lipid interactions are required to maintain the leaflet asymmetry needed to stabilize unilamellar vesicles over flat membranes (Safran et al. 1990). Molecular dynamics simulations have furthermore confirmed very weak leaflet 
A. Callan-Jones et al.

asymmetry in two-component spherical vesicles (Cooke and Deserno 2006); they concluded that transverse sorting in vesicles on the scale of $50 \mathrm{~nm}$ is negligible unless some other physico-chemical agent is involved, such as lipid-lipid interactions.

\section{PROTEIN BINDING ENHANCES LIPID SORTING}

Membrane-bound proteins interact differently with the various lipid species in the membrane and thus can strongly influence lipid distributions. First, when a protein binds to multiple lipid receptors, it clusters these lipids; the resulting cluster generally has a preferred curvature and thus can be sorted into or out of highly curved membrane regions. Second, proteins can interact indirectly with different lipid species based on weaker, nonspecific interactions between protein receptors and other lipids. This can also lead to changes in lipid distribution, depending on the membrane curvature.

As mentioned earlier, curvature-induced lipid sorting is generally quite weak because of the small size of the lipids. The area of a protein is typically an order of magnitude or more greater than that of a lipid. As a result, even at low concentrations, because of their shape membrane-bound and transmembrane proteins are quite easily sorted by curvature. This effect was first studied theoretically by Leibler (1986) and subsequently by Netz and Pincus (1995), Kralj-Iglic et al. (1996), and Ramaswamy et al. (2000). Analogous to lipids sorted based on spontaneous curvature, a protein has a preferred curvature and when bound or inserted, it will locally deform the membrane (see, for instance, Tsafrir et al. 2001; Campelo et al. 2008; Auth and Gompper 2009); this energy will be minimized if the membrane curvature matches that of the protein. As a result, because of this curvature-concentration coupling, the bending energy of a tube pulled from a vesicle is reduced by either excluding or enriching the tube in proteins, depending on their intrinsic shape. This leads to a reduction of the bending stiffness of the membrane. In fact, this has been measured for conical-shaped transmembrane proteins such as calcium ATP-ase (Girard et al. 2005), whereas no reduction of the bending stiffness was measured for the cylindrical shaped protein pump bacteriorhodopsin (Manneville et al. 1999). Confocal microscopy of membrane nanotubes has also revealed the sorting of the curvaturesensitive GTP-ase ArfGap1 (Ambroggio et al. 2010). In addition, cholera (Sorre et al. 2009; Tian and Baumgart 2009) and Shiga toxins (Safouane et al. 2010) bound to DOPC membranes with small amounts of the toxin lipid receptor have been observed to be strongly excluded from tubes. Both types of toxin prefer negative curvature, whereas tubes have positive curvature, and hence on energetic grounds should be excluded from tubes when bound to the outer leaflet. Although sorting of cholera toxin was observed (Sorre et al. 2009; Tian and Baumgart 2009), because of the low concentration no concomitant effect was detected on the bending stiffness (Sorre et al. 2009). Thus, on the one hand, the specific binding of curvature-sensitive proteins to certain lipids can be seen as a means to selectively sort these lipids. On the other, proteins can indirectly aid in sorting of lipids that are not bound to the protein, if the membrane composition is near phase separation, as discussed below.

The clustering of some lipids, such as the binding of cholera toxin to GM1 (Ribi et al. 1988) or of Shiga toxin to Gb3 (Ling et al. 1998), may have an entropic effect on the sorting of other lipids in the membrane, such as that of sphingolipids discussed earlier. Generally, clustering a certain type of lipid by a protein or even actin filaments can induce phase separation in a homogeneous membrane even if this component is present in a very small quantity (Hammond et al. 2005; Liu and Fletcher 2006; Römer et al. 2010) and (Safouane et al. 2010). The effects of protein on the phase behavior of lipid membrane are an interesting phenomenon and can be understood as follows. Consider a homogeneous ternary mixture of SM, DOPC, and cholesterol in the $\mathrm{L}_{\mathrm{d}}$-phase, with a very small percentage of GM1. Because of lipidlipid interactions, SM prefers to aggregate with itself or with cholesterol, and repels DOPC. 
For compositions outside the coexistence region, entropy prevents this segregation. GM1 interacts favorably with sphingomyelin. For a fixed ratio of SM, cholesterol, and DOPC, adding a small amount of GM1 can slightly tip the balance of entropy versus phase separation-causing interactions in favor of interactions. Furthermore, if GM1 is clustered together, the cost of entropy in segregating lipid species is lower. Thus, clustered GM1 can bring the membrane closer to phase separation. This has the effect of making the membrane more susceptible to curvature-induced lipid sorting.

These effects were actually observed by Sorre et al. (2009), wherein a small percentage of GM1 lipids were clustered together by the pentameric B-subunit of cholera toxin bound to the outer leaflet of the membrane. In this case, for membrane compositions near phase separation, the mechanical quantity $\alpha$, measuring the effect of sphingolipid sorting on the force, was found to increase substantially in magnitude on cholera toxin binding, consistent with the protein bringing the membrane composition even closer to phase separation. It should be noted that the curvature dependence of cholera toxin might also affect the lipid sorting and the pulling force. Yet, the results of Sorre et al. showed that the dominant effect of cholera toxin binding was to enhance the susceptibility for sorting, consistent with studies showing that a small amount of cholera toxin bound to homogeneous vesicles can trigger phase separation (see above).

This indirect effect of proteins on lipid organization may be relevant to plasma membranes, where it has been observed that the formation of macroscopic domains on plasma membrane spheres is triggered by specific protein binding (Lingwood et al. 2008). Additionally, in vitro experiments on reconstituted COPI-coated vesicles indicated that the membrane compositions underneath coated regions were enriched in unsaturated lipids compared with the bare regions (Manneville et al. 2008) in agreement with (Brugger et al. 2000). Very generally, one might expect that in vivo clustering of lipids or of proteins can shift the membrane composition close to phase separation, thus aiding in lipid sorting and raft formation.
Up to now, we have discussed sorting mechanisms that may explain the decrease of sphingolipid concentration in transport intermediates along the retrograde pathway. It fails, however, to account for the observed enrichment of these lipids between the TGN and the plasma membrane. We discuss briefly how protein may play a key role in concentrating sphingolipids into highly curved transport intermediates. In Safouane et al. (2010), tubular invaginations were induced on GUVs by the binding of the B-subunit of Shiga toxin to its lipid receptor Gb3. Using different fluorophores, it was found that the concentration of sphingolipids in the tubes increased as the membrane composition approached that of phase separation. This finding was rather striking, given that earlier, sphingolipids were found to be depleted in tubes (see above). This observation was explained by noting that sphingolipids have favorable interactions with Gb3 relative to those between DOPC and Gb3. These interactions were found to be strong enough so that sphingolipids were essentially "dragged" along with the curvature-sorted Gb3-Shiga complexes, outweighing the increase in bending stiffness of the tube caused by the sphingolipid enrichment, in line with (Klemm et al. 2009).

\section{CONCLUDING REMARKS}

In cell membranes, it is very unlikely that individual lipids, unassisted by interactions with themselves or with proteins, can be enriched in curved regions simply based on their shape alone. Rather, larger-scale effects, resulting from lipid-lipid interactions or from lipidprotein interactions, appear to be essential in making the membrane susceptible to curvaturedriven sorting (see Fig. 4 for a cartoon summarizing the different sorting cases). An interesting corollary is that the direction of lipid sorting, into or out of highly curved vesicles, may be dependent on the type of protein that interacts with it either directly or indirectly. This is very different from protein cargoes that are known to be selected and transported by specific ligand-receptor interactions with membrane-bound proteins. In the case of 
A. Callan-Jones et al.

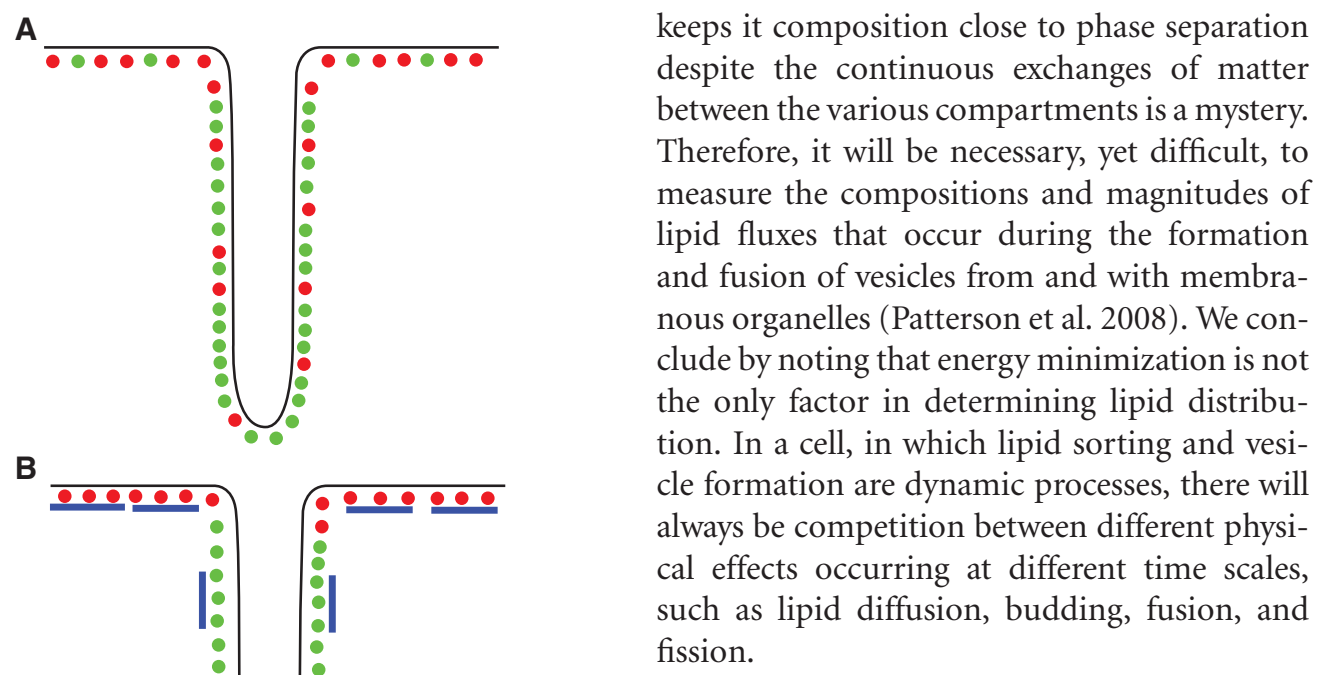

\section{ACKNOWLEDGMENTS}

We are grateful to Gil Toombes, Bruno Goud, J.B. Manneville, J.F. Joanny, J. Prost, and G. van Meer for constructive discussions. We sincerely thank our collaborators Mahassine Safouane, Ludwig Berland, Aurélien Roux, who have been deeply involved in some of the work that was discussed here. We also thank Gil Toombes for carefully reading the manuscript. This work was supported by the European Commission (NoE SoftComp) and the Human Frontier Science Program Organisation. The group belongs to the French research consortium "CellTiss." B.S. has been supported by a grant from the Direction Générale pour l'Armement and by the Fondation pour la Recherche Médicale, A.C.-J by the Association pour la Recherche contre le Cancer and by the NoE SoftComp. for negative curvature (blue) interacting with saturated lipids (red) on the outside. The protein is depleted from the tube and assists in further excluding saturated lipids. $(C)$ When the protein is on the other side, saturated lipids are enriched in the tube.

lipids, protein-aided transport is mediated by weaker nonspecific interactions of longer range. It seems crucial, although, that the membrane composition be close to phase separation in order for protein-induced redistribution to be effective. Many open questions remain, however. For instance, how the cell membrane

\section{REFERENCES}

Ambroggio E, Sorre B, Bassereau P, Goud B, Manneville J-B, Antonny B. 2010. ArfGAP1 generates an Arf1 gradient on continuous lipid membranes displaying flat and curved regions. EMBO J 29: 292-303.

Antonny B. 2006. Membrane deformation by protein coats. Curr Opin Cell Biol 18: 386-394.

Auth T, Gompper G. 2009. Budding and vesiculation induced by conical membrane inclusions. Phys Rev E 80: 031901-031910.

Bagatolli LA, Gratton E. 2000. Two photon fluorescence microscopy of coexisting lipid domains in giant unilamellar 
vesicles of binary phospholipid mixtures. Biophys J 78: 290-305.

Barlowe C. 2000. Traffic COPs of the early secretory pathway. Traffic 1: 371-377.

Baumgart T, Hess ST, Webb WW. 2003. Imaging coexisting fluid domains in biomembrane models coupling curvature and line tension. Nature 425: 821-824.

Brugger B, Sandhoff R, Wegehingel S, Gorgas K, Malsam J, Helms JB, Lehmann WD, Nickel W, Wieland FT. 2000. Evidence for segregation of sphingomyelin and cholesterol during formation of COPI-coated vesicles. $\mathrm{J} \mathrm{Cell}$ Biol 151: 507-518.

Campelo F, McMahon HT, Kozlov MM. 2008. The hydrophobic insertion mechanism of membrane curvature generation by proteins. Biophys J 95: 2325-2339.

Canham PB. 1970. Minimum energy of bending as a possible explanation of biconcave shape of human red blood cell. J Theor Biol 26: 61-81.

Connell SD, Smith DA. 2006. The atomic force microscope as a tool for studying phase separation in lipid membranes (Review). Mol Membr Biol 23: 17-28.

Cooke IR, Deserno M. 2006. Coupling between lipid shape and membrane curvature. Biophys J 91: 487-495.

Cosson P, Letourneur F. 1994. Coatomer interaction with di-lysine endoplasmic-reticulum retention motifs. Science 263: 1629-1631.

Cuvelier D, Derényi I, Bassereau P, Nassoy P. 2005. Coalescence of membrane tethers: experiments, analysis and applications. Biophys J 88: 2714-2726.

de Almeida RFM, Fedorov A, Prieto M. 2003. Sphingomyelin/phosphatidylcholine/ cholesterol phase diagram: Boundaries and composition of lipid rafts. Biophys J 85: 2406-2416.

Derényi I, Jülicher F, Prost J. 2002. Formation and interaction of membrane tubes. Phys Rev Lett 88: 238101.

Derganc J. 2007. Curvature-driven lateral segregation of membrane constituents in Golgi cisternae. Phys Biol 4: 317-324.

Dietrich C, Bagatolli LA, Volovyk ZN, Thompson NL, Levi M, Jacobson K, Gratton E. 2001. Lipid rafts reconstituted in model membranes. Biophys J 80: 1417-1428.

Ehrlich M, Boll W, van Oijen A, Hariharan R, Chandran K, Nibert ML, Kirchhausen T. 2004. Endocytosis by random initiation and stabilization of clathrin-coated pits. Cell 118: 591-605.

Evans E. 1974. Bending resistance and chemically induced moments in membrane bilayers. Biophys J 14: 923-931.

Evans E, Yeung A. 1994. Hidden dynamics in rapid changes of bilayer shape. Chem Phys Lipids 73: 39-56.

Girard P, Prost J, Bassereau P. 2005. Passive or active fluctuations in membranes containing proteins. Phys Rev Lett 94: 088102.

Goni FM, Alonso A, Bagatolli LA, Brown RE, Marsh D, Prieto M, Thewalt JL. 2008. Phase diagrams of lipid mixtures relevant to the study of membrane rafts. Biochim Biophys Acta 1781: 665-684.

Gruenberg J. 2003. Lipids in endocytic membrane transport and sorting. Curr Opin Cell Biol 15: 382.

Gruner SM. 1989. Stability of lyotropic phases with curved interfaces. J Phys Chem 93: 7562-7570.
Hammond AT, Heberle FA, Baumgart T, Holowka D, Baird B, Feigenson GW. 2005. Crosslinking a lipid raft component triggers liquid ordered-liquid disordered phase separation in model plasma membranes. Proc Natl Acad Sci 102: $6320-6325$.

Helfrich W. 1973. Elastic properties of lipid bilayers: theory and possible experiments. $Z$ Naturforsch Sect C J Biosci 28: $693-703$.

Hochmuth RM, Evans EA. 1982. Extensional flow of erythrocyte membrane from cell body to elastic tether. I. Analysis. Biophys J 39: 71-81.

Jiang H, Powers TR. 2008. Curvature-driven lipid sorting in a membrane tubule. Phys Rev Lett 101: 018103.

Kamal MM, Mills D, Grzybek M, Howard J. 2009. Measurement of the membrane curvature preference of phospholipids reveals only weak coupling between lipid shape and leaflet curvature. Proc Natl Acad Sci 106: 22245-22250.

Klemm RW, Ejsing CS, Surma MA, Kaiser HJ, Gerl MJ, Sampaio JL, de Robillard Q, Ferguson C, Proszynski TJ, Shevchenko A, et al. 2009. Segregation of sphingolipids and sterols during formation of secretory vesicles at the transGolgi network. J Cell Biol 185: 601-612.

Kozlov MM, Helfrich W. 1992. Effects of a cosurfactant on the stretching and bending elasticities-a surfactant monolayer. Langmuir 8: 2792-2797.

Kralj-Iglic V, Svetina S, Zeks B. 1996. Shapes of bilayer vesicles with membrane embedded molecules. Eur Biophys J 24: 311-321.

Lawrence JC, Saslowsky DE, Edwardson JM, Henderson RM. 2003. Real-time analysis of the effects of cholesterol on lipid raft behavior using atomic force microscopy. Biophys J 84: 1827-1832.

Leduc C, Campas O, Zeldovich K, Roux A, Jolimaitre P, Bourel-Bonnet L, Goud B, Joanny JF, Bassereau P, Prost J. 2004. Cooperative extraction of membrane nanotubes by molecular motors. Proc Natl Acad Sci 101: 1709617101.

Leibler S. 1986. Curvature instability in membranes. J Phys 47: 507-516.

Ling H, Boodhoo A, Hazes B, Cummings MD, Armstrong GD, Brunton JL, Read RJ. 1998. Structure of the shigalike toxin I B-pentamer complexed with an analogue of its receptor Gb3. Biochemistry 37: 1777-1788.

Lingwood D, Ries J, Schwille P, Simons K. 2008. Plasma membranes are poised for activation of raft phase coalescence at physiological temperature. Proc Natl Acad Sci 105: 10005-10010.

Lingwood D, Simons K. 2010. Lipid rafts as a membraneorganizing principle. Science 327: 46-50.

Liu AP, Fletcher DA. 2006. Actin polymerization serves as a membrane domain switch in model lipid bilayers. Biophys J 91: 4064-4070.

Lodish H, Berk A, Matsudaira P, Kaiser CA, Krieger M, Scott MP, Zipursky L, Darnell J. 2003. Molecular Cell Biology, 5th ed. W.H Freeman, New York.

Manneville J-B, Bassereau P, Levy D, Prost J. 1999. Activity of transmembrane proteins induces magnification of shape fluctuations of lipid membranes. Phys Rev Lett 82: 4356-4359.

Manneville J-B, Casella JF, Ambroggio E, Gounon P, Bertherat J, Bassereau P, Cartaud J, Antonny B, Goud B. 
A. Callan-Jones et al.

2008. COPI coat assembly occurs on liquid disordered domains and the associated membrane deformations are limited by membrane tension. Proc Natl Acad Sci 105: $16946-16951$.

Markin VS. 1981. Lateral organization of membranes and cell shapes. Biophys J 36: 1-19.

Marsh D. 2006. Elastic curvature constants of lipid monolayers and bilayers. Chem Phys Lipids 144: 146-159.

Maxfield FR, McGraw TE. 2004. Endocytic recycling. Nat Rev Mol Cell Biol 5: 121.

McConnell HM, Vrljic M. 2003. Liquid-liquid immiscibility in membranes. Ann Rev Biophys Biomol Struct 32: 469492.

Mukherjee S, Maxfield FR. 2000. Role of membrane organization and membrane domains in endocytic lipid trafficking. Traffic 1: 203-211.

Mukherjee S, Soe TT, Maxfield FR. 1999. Endocytic sorting of lipid analogues differing solely in the chemistry of their hydrophobic tails. J Cell Biol 144: 1271-1284.

Mukhopadhyay R, Huang KC, Wingreen NS. 2008. Lipid localization in bacterial cells through curvature-mediated microphase separation. Biophys J 95: 1034-1049.

Netz RR, Pincus P. 1995. Inhomogeneous fluid membranes: Segregation, ordering, and effective rigidity. Phys Rev E 52: $4114-4128$.

Pan J, Mills TT, Tristram-Nagle S, Nagle JF. 2008. Cholesterol perturbs lipid bilayers nonuniversally. Phys Rev Lett 100: 198103-198104.

Parthasarathy R, Yu C-h, Groves JT. 2006. Curvaturemodulated phase separation in lipid bilayer membranes. Langmuir 22: 5095-5099.

Patterson GH, Hirschberg K, Polishchuk RS, Gerlich D Phair RD, Lippincott-Schwartz J. 2008. Transport through the Golgi apparatus by rapid partitioning within a two-phase membrane system. Cell 133: 1055-1067.

Powers TR, Huber G, Goldstein RE. 2002. Fluid-membrane tethers: Minimal surfaces and elastic boundary layers. Phys Rev E 65: 041901.

Ramaswamy S, Prost J, Toner J. 2000. Nonequilibrium fluctuations, travelling waves, and instabilities in active membranes. Phys Rev Lett 84: 3494-3497.

Rawicz W, Olbrich KC, McIntosh T, Needham D, Evans E. 2000. Effect of chain length and unsaturation on elasticity of lipid bilayers. Biophys J 79: 328-339.

Rawicz W, Smith BA, McIntosh TJ, Simon SA, Evans E. 2008. Elasticity, strength, and water permeability of bilayers that contain raft microdomain-forming lipids. Biophys J 94: 4725-4736.

Reeves D, Ursell T, Sens P, Kondev J, Phillips R. 2008. Membrane mechanics as a probe of ion-channel gating mechanisms. Phys Rev E 78: 041901.

Ribi HO, Ludwig DS, Mercer KL, Schoolnik GK, Kornberg RD. 1988. Three-dimensional structure of cholera toxin penetrating a lipid membrane. Science 239: 1272-1276.

Römer W, Pontani L-L, Sorre B, Rentero C, Berland L, Chambon V, Lamaze C, Bassereau P, Sykes C, Gaus K et al. 2010. Actin dynamics drive membrane reorganization and scission in clathrin independent endocytosis. Cell 140: 540-553.
Roux A, Cuvelier D, Nassoy P, Prost J, Bassereau P, Goud B. 2005. Role of curvature and phase transition in lipid sorting and fission of membrane tubules. EMBO $\mathrm{J} \mathbf{2 4}$ 1537-1545.

Safouane M, Berland L, Callan-Jones A, Sorre B, Römer W, Johannes L, Toombes GES, Bassereau P. 2010. Lipid cosorting mediated by Shiga Toxin-induced tubulation. Traffic 11: 1519-1529.

Safran SA, Pincus P, Andelman D. 1990. Theory of spontaneous vesicle formation in surfactant mixtures. Science 248: $354-356$.

Safran SA, Pincus PA, Andelman D, Mackintosh FC. 1991. Stability and phase-behavior of mixed surfactant vesicles. Phys Rev A 43: 1071-1078.

Seifert U. 1993. Curvature-induced lateral phase segregation in two-component vesicles. Phys Rev Lett 70: 1335-1338.

Simons K, Ikonen E. 1997. Functional rafts in cell membranes. Nature 387: 569-572.

Simons K, Van Meer G. 1988. Lipid sorting in epithelialcells. Biochemistry 27: 6197-6202.

Sorre B, Callan-Jones A, Manneville J-B, Nassoy P, Joanny JF, Prost J, Goud B, Bassereau P. 2009. Curvature-driven lipid sorting needs proximity to a demixing point and is aided by proteins. Proc Natl Acad Sci 106: 5622-5626.

Stamnes MA, Craighead MW, Hoe MH, Lampen N, Geromanos S, Tempst P, Rothman JE. 1995. An integral membrane component of coatomer-coated transport vesicles defines a family of proteins involved in budding. Proc Natl Acad Sci 92: 8011-8015.

Szleifer I, Kramer D, Benshaul A, Roux D, Gelbart WM. 1988. Curvature elasticity of pure and mixed surfactant films. Phys Rev Lett 60: 1966-1969.

Tian A, Baumgart T. 2009. Sorting of lipids and proteins in membrane curvature gradients. Biophys J 96: 26762688.

Tian A, Capraro BR, Esposito C, Baumgart T. 2009. Bending stiffness depends on curvature of ternary lipid mixture tubular membranes. Biophys J 97: 1636-1646.

Tsafrir I, Sagi D, Arzi T, Guedeau-Boudeville M-A, Frette V, Kandel D, Stavans J. 2001. Pearling Instabilities of Membrane Tubes with Anchored Polymers. Phys Rev Lett 86: 1138-1141.

van Meer G, Lisman Q. 2002. Sphingolipid transport: rafts and translocators. J Biol Chem 277: 25855-25858.

van Meer G, Sprong H. 2004. Membrane lipids and vesicular traffic. Curr Opin Cell Biol 16: 373-378.

van Meer G, Voelker DR, Feigenson GW. 2008. Membrane lipids: where they are and how they behave. Nat Rev Mol Cell Biol 9: 112-124.

Veatch SL, Keller SL. 2002. Organization in lipid membranes containing cholesterol. Phys Rev Lett 89: 268101.

Waugh R, Evans EA. 1979. Thermoelasticity of red bloodcell membrane. Biophys J 26: 115-131.

Waugh RE, Song J, Svetina S, Zeks B. 1992. Local and non local curvature elasticity in bilayer membranes by tether formation from lecithin vesicles. Biophys J 61: 974-982.

Yuan C, Furlong J, Burgos P, Johnston LJ. 2002. The size of lipid rafts: an atomic force microscopy study of ganglioside GM1 domains in sphingomyelin/DOPC/ cholesterol membranes. Biophys J 82: 2526-2535. 


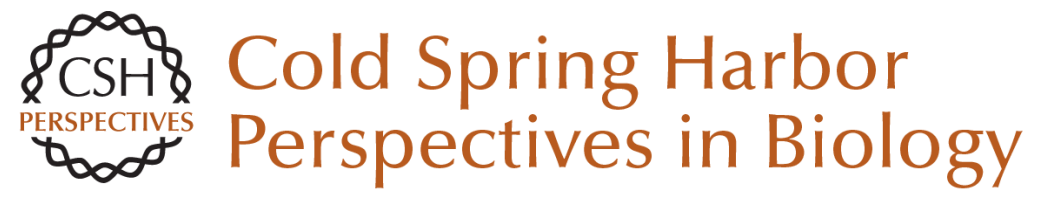

\section{Curvature-Driven Lipid Sorting in Biomembranes}

Andrew Callan-Jones, Benoit Sorre and Patricia Bassereau

Cold Spring Harb Perspect Biol 2011; doi: 10.1101/cshperspect.a004648 originally published online January 12, 2011

\section{Subject Collection The Biology of Lipids}

Role of Lipids in Virus Replication Maier Lorizate and Hans-Georg Kräusslich

Model Answers to Lipid Membrane Questions Ole G. Mouritsen

\section{Glycosphingolipid Functions} Clifford A. Lingwood

Regulation of Cholesterol and Fatty Acid Synthesis

Jin Ye and Russell A. DeBose-Boyd

\section{Lipid-Mediated Endocytosis}

Helge Ewers and Ari Helenius

\section{Fluorescence Techniques to Study Lipid}

Dynamics

Erdinc Sezgin and Petra Schwille

Lysosomal Lipid Storage Diseases Heike Schulze and Konrad Sandhoff

\section{Distribution and Functions of Sterols and} Sphingolipids

J. Thomas Hannich, Kyohei Umebayashi and Howard Riezman
Membrane Organization and Lipid Rafts Kai Simons and Julio L. Sampaio

Shotgun Lipidomics on High Resolution Mass

Spectrometers

Dominik Schwudke, Kai Schuhmann, Ronny

Herzog, et al.

Glycosphingolipid Functions Clifford A. Lingwood

Phosphoinositides in Cell Architecture Annette Shewan, Dennis J. Eastburn and Keith Mostov

Synthesis and Biosynthetic Trafficking of Membrane Lipids

Tomas Blom, Pentti Somerharju and Elina Ikonen

Lipid Polymorphisms and Membrane Shape Vadim A. Frolov, Anna V. Shnyrova and Joshua Zimmerberg

Specificity of Intramembrane Protein-Lipid Interactions

Francesc-Xabier Contreras, Andreas Max Ernst, Felix Wieland, et al.

Dynamic Transbilayer Lipid Asymmetry Gerrit van Meer

For additional articles in this collection, see http://cshperspectives.cshlp.org/cgi/collection/

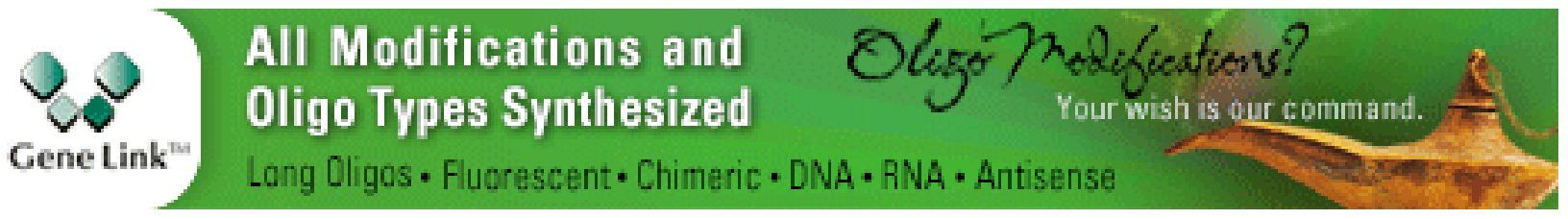

Copyright @ 2011 Cold Spring Harbor Laboratory Press; all rights reserved 